\title{
Results of beetles (Coleoptera) survey of Zástudánčí National Nature Reserve (Central Moravia) 2008 - part 2
}

Oto Nakládal

Výsledky průzkumu brouků (Coleoptera) NPR Zástudánčí (stř̌ední Morava) provedeného v roce 2008 - 2. část. - Čas. Slez. Muz. Opava (A), 60: 165-178, 2011.

Abstract: Faunistic survey of beetles (Coleoptera) in Zástudánčí National Nature Reserve (Central Moravia) was performed in 2008. Various survey and trapping methods were used in order to record as many species of beetles. The survey recorded 857 species from 64 families. Faunistic data of 41 of them (Nosodendridae, Dermestidae, Anobidae, Cleridae, Melyridae, Sphindidae, Kateretidae, Nitidulidae, Monotomidae, Silvanidae, Cucujidae, Laemophloeidae, Phalacridae, Cryptophagidae, Erotylidae, Byturidae, Bothrideridae, Cerylonidae, Endomychidae, Coccinellidae, Zopheridae, Corylophidae, Latridiidae, Mycetophagidae, Ciidae, Tetratomidae, Melandryidae, Mordellidae, Tenebrionidae, Oedemeridae, Pyrochroidae, Salpingidae, Anthicidae, Scraptiidae, Cerambycidae, Orsodacnidae, Chrysomelidae, Anthribidae, Attelabidae, Apionidae, and Curculionidae) are presented. Presenting data represent 1394 specimens belonging to 401 species. Cucujus cinnaberinus (Cucujidae), species belonging to the NATURA 2000, was recorded.

Key words: faunistics, Coleoptera, Zástudánčí National Nature Reserve, Czech Republic, Cucujus cinnaberinus

\section{Introduction}

Zástudánčí National Nature Reserve is at first sight very interesting territory from fauna diversity point of view. Despite of this fact, it was very insufficiently documented.

Inventory survey of aquatic beetles was made by Merta (2004), butterflies with day activity by Čelechvoský (1993, 2001a,b) and Čelechovská (2001). First integral data about beetles (Coleoptera) brings Nakládal (2011) and this paper which form its second published part.

Natural conditions and characteristic of the study area have been summarized by Nakládal (2011) in first published part of the survey.

\section{Material and methods}

The aim of this survey was to record as many species of beetles, therefore various collecting methods were utilized, including individual sampling (especially in the riparian zone of the Morava river and aquatic habitats), beating of trees and bushes, sweeping (in day and also at night), capturing of aquatic beetles by sieve, pitfall trapping, examination of excrements and carrion, sifting of organic substrates (especially from anthills and tree hollows and bases), night sampling from trees, luring at light on white canvas, capturing on fermented tree sap, luring by ferment fruits, collecting trunks and branches and their subsequent getting under laboratory condition.

The study area was visited on 9.v., 29.v., 14.vi., 22.vi., 11.vii., 17.viii. and 19.x.2008. The pitfall traps (ethylenglycol was utilized to fix the specimens) were set on 29.v.-11.vii.2008 and 11.vii. -17.viii.2008 (to the 4 different biotopes - see Nakládal (2011) for exact position of the traps).

The nomenclature follows Löbel \& Smetana (2007, 2008, 2010) excluding Curculionoidea. System of families in Curculionoidea superfamily follows Vorst (2010). The species within each family are listed alphabetically.

Identification: Family Dermestidae identified Jiří Háva (Únětice), Anobidae Petr Zahradník (Praha), Kateretidae, Nitidulidae and Byturidae Josef Jelínek (Praha), Monotomidae, Silvanidae, Laemophloeidae, Cryptophagidae, Erotylidae, Cerylonidae, Corylophidae, Latridiidae, Mycetophagidae, Ciidae and Salpingidae Pavel Průdek (Brno), Phalacridae Zdeněk Švec (Praha), Coccinellidae Vladimír Navrátil (Praha), Melandryidae, Mordellidae and Scraptiidae Jan Horák (Praha), Oedemeridae (Vladimír Švihla), Anthicidae Zbyněk Kejval (Chodsko Museum, Domažlice), Orsodacnidae, Chrysomelidae, Anthribidae, Attelabidae, Apionidae, and Curculionidae (except Scolytinae and Platypodinae) Jaromír Strejček (Praha) - unless otherwise stated in the Results. Others families were identified by author. 


\section{Results}

Faunistics data for 41 families are presented in this part of paper (Nosodendridae, Dermestidae, Anobidae, Cleridae, Melyridae, Sphindidae, Kateretidae, Nitidulidae, Monotomidae, Silvanidae, Cucujidae, Laemophloeidae, Phalacridae, Cryptophagidae, Erotylidae, Byturidae, Bothrideridae, Cerylonidae, Endomychidae, Coccinellidae, Zopheridae, Corylophidae, Latridiidae, Mycetophagidae, Ciidae, Tetratomidae, Melandryidae, Mordellidae, Tenebrionidae, Oedemeridae, Pyrochroidae, Salpingidae, Anthicidae, Scraptiidae, Cerambycidae, Orsodacnidae, Chrysomelidae, Anthribidae, Attelabidae, Apionidae and Curculionidae).

The families contain records of 1394 speciment representing 401 species (from all count of 3585 spec. belonging to 857 species from 64 families).

Data „Tovačov env., Zástudánčí National Nature Reserve“ belong to all recorded specimens.

All specimens were captured by the author and they are deposited in his private collection (unless otherwise stated in the Results).

Abbreviations: PFT - pitfall trap

\section{NOSODENDRIDAE}

Nosodendron fasciculare (Olivier, 1790): (6569), 9.v.2008, on oozing sap of Acer, 1 spec.; (6569), 14.vi.2008, on oozing sap of Quercus, 1 spec.

\section{DERMESTIDAE}

Anthrenus scrophulariae scrophulariae (Linnaeus, 1758): (6569), 14.vi.2008, 1 spec.

Dermestes frischi Kugelann, 1792: (6569), 22.vi.2008, 1 spec.; (6569), 11.vii.2008, 1 spec.

Dermestes murinus murinus Linnaeus, 1758: (6569), 22.vi.2008, on carrion, 1 spec.; (6569), 11.vii.17.viii.2008, PFT - locality 3, 2 spec.; (6569), 17.viii.2008, 1 spec.

Globicornis nigripes (Fabricius, 1792): (6569), 29.v.2008, 1 spec.

Trinodes hirtus (Fabricius, 1781): (6569), 14.vi.2008, 1 spec., O. Nakládal det.; (6569), 22.vi.2008, sifted from base of old tree, 1 spec., O. Nakládal det.

Trogoderma glabrum (Herbst, 1783): (6569), 22.vi.2008, 1 spec.

\section{PTINIDAE}

Cacotemnus rufipes (Fabricius, 1792): (6569), 22.vi.2008, 1 spec.

Gastrallus laevigatus (Olivier, 1790): (6569), 11.vii.2008, 1 spec.

Hemicoelus fulvicornis (Sturm, 1837): (6569), 14.vi.2008, 1 spec.; (6569), 22.vi.2008, 1 spec.

Oligomerus brunneus (Olivier, 1790): (6569), 11.vii.2008, 1 spec.

Ptilinus pectinicornis (Linnaeus, 1758): (6569), 29.v.2008, 1 spec.

Ptinomorphus imperialis (Linnaeus, 1767): (6569), 9.v.2008, 4 spec.; (6569), 22.vi.2008, 1 spec.; (6669), 9.v.2008, 1 spec.

Ptinus pilosus P. W. J. Müller, 1821: (6569), 22.vi.2008, sifted from base of old tree, 1 spec.; (6569), 19.x.2008, 4 spec.

Ptinus rufipes Olivier, 1790: (6569), 29.v.2008, 7 spec.; (6569), 14.vi.2008, 1 spec.; (6569), 22.vi.2008, 1 spec. Xyletinus ater (Creutzer, 1796): (6569), 22.vi.2008, 1 spec.

\section{CLERIDAE}

Thanasimus formicarius formicarius (Linnaeus, 1758): (6569), 9.v.2008, 1 spec.

\section{MELYRIDAE}

Axinotarsus marginalis (Laporte de Castelnau, 1840): (6569), 14.vi.2008, 1 spec.; (6669), 22.vi.2008, 1 spec.

Axinotarsus pulicarius (Fabricius, 1775): (6569), 11.vii.2008, 1 spec.

Axinotarsus ruficollis (Olivier, 1790): (6569), 11.vii.2008, 1 spec.

Charopus concolor (Fabricius, 1801): (6669), 22.vi.2008, 1 spec.

Charopus flavipes (Paykull, 1798): (6569), 22.vi.2008, 2 spec.; (6669), 22.vi.2008, 1 spec.

Cordylepherus viridis (Fabricius, 1787): (6669), 22.vi.2008, 2 spec.

Dasytes aeratus Stephens, 1829: (6569), 9.v.2008, 2 spec., O. Nakládal det.

Dasytes fusculus (Illiger, 1801): (6569), 9.v.2008, 1 spec., O. Nakládal det. 
Dasytes plumbeus (O.F. Müller, 1776): (6569), 29.v.2008, 1 spec., O. Nakládal det.

Malachius bipustulatus (Linnaeus, 1758): (6569), 29.v.2008, 3 spec.; (6569), 14.vi.2008, 1 spec.

Trichoceble floralis (A. G. Olivier, 1790): (6569), 9.v.2008, 1 spec., O. Nakládal det.

\section{SPHINDIDAE}

Aspidiphorus orbiculatus (Gyllenhal, 1808): (6569), 14.vi.2008, 1 spec.

\section{KATERETIDAE}

Brachypterolus linariae (Stephens, 1830): (6569), 9.v.2008, 1 spec.

Brachypterus urticae (Fabricius, 1792): (6569), 14.vi.2008, 4 spec.; (6569), 22.vi.2008, 7 spec.

Kateretes pusillus (Thunberg, 1794): (6569), 9.v.2008, 1 spec.

\section{NITIDULIDAE}

Carpophilus hemipterus (Linnaeus, 1758): (6569), 17.viii.2008, 1 spec.

Carpophilus marginellus Motschulsky, 1858: (6569), 17.viii.2008, $1 \mathrm{spec.}$

Cychramus luteus (Fabricius, 1787): (6569), 14.vi.2008, 4 spec.

Epuraea aestiva (Linnaeus, 1758): (6569), 9.v.2008, 5 spec.; (6569), 29.v.2008, 4 spec.

Epuraea longula Erichson, 1845: (6569), 9.v.2008, 1 spec.

Epuraea melanocephala (Marsham, 1802): (6569), 9.v.2008, 5 spec.; (6569), 14.vi.2008, 3 spec.; (6569), 22.vi.2008, 1 spec.

Epuraea neglecta (Heer, 1841): (6569), 9.v.2008, 1 spec.

Epuraea pallescens (Stephens, 1835): (6569), 9.v.2008, 2 spec.; (6569), 29.v.2008, 1 spec.

Epuraea unicolor (Olivier, 1790): (6569), 9.v.2008, 2 spec.; (6569), 29.v.2008, 1 spec.; (6569), 14.vi.2008, 3 spec.; (6569), 19.x.2008, 1 spec.

Glischrochilus quadrisignatus (Say, 1835): (6569), 29.v.2008, 1 spec.; (6569), 14.vi.2008, 1 spec.; (6569), 17.viii.2008, 1 spec.; (6569), 19.x.2008, 17 spec.

Meligethes aeneus (Fabricius, 1775): (6569), 14.vi.2008, 6 spec.; (6569), 19.x.2008, 1 spec.; (6669), 22.vi.2008, 3 spec.

Meligethes bidens C. Brisout de Barneville, 1863: (6669), 22.vi.2008, 1 spec.

Meligethes difficilis (Heer, 1841): (6569), 29.v.2008, 3 spec.; (6569), 14.vi.2008, 3 spec.

Meligethes egenus Erichson, 1845: (6569), 29.v.2008, 1 spec.

Meligethes flavimanus Stephens, 1830: (6669), 9.v.2008, 1 spec.

Meligethes morosus Erichson, 1845: (6569), 9.v.2008, 3 spec.; (6569), 29.v.2008, 1 spec.; (6569), 14.vi.2008, 1 spec.

Meligethes ovatus Sturm, 1845: (6569), 14.vi.2008, 1 spec.

Meligethes planiusculus (Heer, 1841): (6569), 14.vi.2008, 1 spec.

Meligethes ruficornis (Marsham, 1802): (6569), 9.v.2008, 1 spec.; (6569), 29.v.2008, 2 spec.

Meligethes symphyti (Heer, 1841): (6569), 9.v.2008, 1 spec.; (6569), 14.vi.2008, 2 spec.; (6669), 9.v.2008, 1 spec.

Meligethes viridescens (Fabricius, 1787): (6569), 29.v.2008, 2 spec.; (6569), 22.vi.2008, 1 spec.

Nitidula carnaria (Schaller, 1783): (6569), 11.vii.2008, 4 spec.

Nitidula rufipes (Linnaeus, 1767): (6569), 11.vii.2008, 1 spec.

Omosita colon (Linnaeus, 1758): (6569), 11.vii.2008, 11 spec.

Omosita discoidea (Fabricius, 1775): (6569), 14.vi.2008, on carrion, 2 spec.

Pocadius adustus Reitter, 1888: (6569), 19.x.2008, 2 spec.

Pocadius ferrugineus (Fabricius, 1775): (6569), 11.vii.2008, 4 spec.

\section{MONOTOMIDAE}

Monotoma bicolor A. et G.B. Villa, 1835: (6569), 17.viii.2008, 9 spec.

Monotoma brevicollis brevicollis Aubé, 1837: (6569), 17.viii.2008, 3 spec.

Monotoma picipes Herbst, 1793: (6569), 22.vi.2008, 1 spec.; (6569), 17.viii.2008, 4 spec.

Monotoma spinicollis Aubé, 1837: (6569), 17.viii.2008, 3 spec.

Rhizophagus bipustulatus (Fabricius, 1792): (6569), 14.vi.2008, 1 spec.; (6569), 19.x.2008, 5 spec.

Rhizophagus picipes (Olivier, 1790): (6569), 14.vi.2008, 1 spec. 


\section{SILVANIDAE}

Silvanus bidentatus (Fabricius, 1792): (6569), 14.vi.2008, 1 spec. Uleiota planatus (Linnaeus, 1761): (6569), 17.viii.2008, 1 spec.

\section{CUCUJIDAE}

Cucujus cinnaberinus (Scopoli, 1763): (6569), 17.viii.2008, 6 spec.; (6569), 19.x.2008, 1 spec. of larva.

\section{LAEMOPHLOEIDAE}

Cryptolestes duplicatus (Waltl, 1839): (6569), 14.vi.2008, 1 spec.

Cryptolestes ferrugineus (Stephens, 1831): (6569), 11.vii.2008, 7 spec.; (6569), 17.viii.2008, $1 \mathrm{spec}$.

Lathropus sepicola (P.W. J. Müller, 1821): (6569), 22.vi.2008, 3 spec.; (6569), 19.x.2008, 1 spec.

\section{PHALACRIDAE}

Olibrus aeneus (Fabricius, 1792): (6569), 9.v.2008, 1 spec.

Phalacrus fimetarius (Fabricius, 1775): (6569), 22.vi.2008, 1 spec.

Stilbus testaceus (Panzer, 1797): (6569), 17.viii.2008, 1 spec.

\section{CRYPTOPHAGIDAE}

Atomaria analis Erichson, 1846: (6569), 19.x.2008, 1 spec.

Atomaria atricapilla Stephens, 1830: (6569), 11.vii.2008, 1 spec.; (6569), 19.x.2008, 4 spec.

Atomaria elongatula Erichson, 1846: (6569), 19.x.2008, 1 spec.

Atomaria fuscata (Schönherr, 1808): (6569), 14.vi.2008, 1 spec.; (6569), 22.vi.2008, 6 spec.; (6569), 19.x.2008, $22 \mathrm{spec}$.

Atomaria gravidula Erichson, 1846: (6569), 11.vii.2008, $1 \mathrm{spec}$.

Atomaria lewisi Reitter, 1877: (6569), 9.v.2008, 1 spec.

Atomaria nigrirostris Stephens, 1830: (6569), 29.v.2008, 3 spec.; (6569), 11.vii.2008, 1 spec.

Atomaria nigriventris Stephens, 1830: (6569), 14.vi.2008, in nest of Lasius fuliginosus, 3 spec.; (6569), 22.vi.2008, sifted from base of old tree, 2 spec.

Cryptophagus pallidus pallidus Sturm, 1845: (6569), 14.vi.2008, in nest of Lasius fuliginosus, 1 spec.; (6569), 22.vi.2008, 2 spec.; (6569), 19.x.2008, 7 spec.

Cryptophagus pubescens Sturm, 1845: (6569), 14.vi.2008, 1 spec.; (6569), 17.viii.2008, 1 spec.

Cryptophagus punctipennis C. N. F. Brisout de Barneville, 1863: (6569), 22.vi.2008, 2 spec.; (6569), 19.x.2008, 6 spec.

Cryptophagus reflexus Rey, 1889: (6569), 22.vi.2008, 2 spec.; (6569), 19.x.2008, 5 spec.

Cryptophagus saginatus Sturm, 1845: (6569), 19.x.2008, 3 spec.

Cryptophagus scutellatus Newman, 1834: (6569), 19.x.2008, 1 spec.

Cryptophagus schmidtii Sturm, 1845: (6569), 29.v.-11.vii.2008, PFT - locality 1, 2 spec.; (6569), 14.vi.2008, 1 spec.

Ephistemus globulus (Paykull, 1798): (6569), 17.viii.2008, 2 spec.; (6569), 19.x.2008, 3 spec.

Ephistemus reitteri Casey, 1900: (6569), 9.v.2008, 1 spec.; (6569), 29.v.2008, 1 spec.; (6569), 22.vi.2008, 2 spec.; (6569), 11.vii.2008, 1 spec.; (6569), 19.x.2008, 3 spec.

Telmatophilus typhae (Fallen, 1802): (6569), 22.vi.2008, 1 spec.; (6569), 11.vii.2008, on light, 1 spec.

\section{EROTYLIDAE}

Dacne bipustulata (Thunberg, 1781): (6569), 11.vii.2008, 1 spec.; (6669), 9.v.2008, 1 spec.

Triplax aenea (Schaller, 1783): (6569), 19.x.2008, 1 spec.

Tritoma bipustulata Fabricius, 1775: (6569), 22.vi.2008, 1 spec., O. Nakládal det.

\section{BYTURIDAE}

Byturus ochraceus (Scriba, 1790): (6569), 14.vi.2008, 2 spec.; (6569), 22.vi.2008, 1 spec. 
Byturus tomentosus (DeGeer, 1774): (6569), 9.v.2008, 2 spec.

\section{BOTHRIDERIDAE}

Oxylaemus cylindricus (Creutzer, 1796): (6569), 22.vi.2008, sifted from base of old tree, 1 spec.; (6569), 19.x.2008, 1 spec.

\section{CERYLONIDAE}

Cerylon deplanatum Gyllenhal, 1827: (6569), 17.viii.2008, 1 spec.

Cerylon fagi C. Brisout de Barneville, 1867: (6569), 19.x.2008, 1 spec.

Cerylon ferrugineum Stephens, 1830: (6569), 19.x.2008, 1 spec.

Cerylon histeroides (Fabricius, 1792): (6569), 11.vii.2008, 1 spec.; (6569), 19.x.2008, 3 spec.

\section{ENDOMYCHIDAE}

Holoparamecus caularum Aubé, 1843: (6569), 17.viii.2008, 1 spec.

Mycetaea subterranea (Fabricius, 1801): (6569), 22.vi.2008, sifted from base of old tree, 1 spec., P. Průdek det.; (6569), 19. x. 2008, 1 spec.

\section{COCCINELLIDAE}

Adalia bipunctata (Linnaeus, 1758): (6569), 9.v.2008, 3 spec.; (6569), 22.vi.2008, 2 spec.; (6569), 11.vii.2008, 2 spec.; (6669), 22.vi.2008, 2 spec.

Adalia decempunctata (Linnaeus, 1758): (6569), 9.v.2008, 3 spec.; (6569), 29.v.2008, 4 spec.; (6569), 14.vi.2008, 1 spec.; (6669), 9.v.2008, 5 spec.

Calvia decemguttata (Linnaeus, 1767): (6569), 14.vi.2008, 1 spec.; (6569), 11.vii.2008, 2 spec.

Calvia quatuordecimguttata (Linnaeus, 1758): (6569), 9.v.2008, 1 spec.; (6569), 29.v.2008, 1 spec.; (6569), 14.vi.2008, 1 spec.

Coccinella quinquepunctata Linnaeus, 1758: (6569), 17.viii.2008, 2 spec.

Coccinella septempunctata septempunctata Linnaeus, 1758: (6569), 9.v.2008, 1 spec.; (6569), 22.vi.2008, 1 spec.; (6569), 11.vii.2008, 1 spec.

Exochomus quadripustulatus (Linnaeus, 1758): (6569), 9.v.2008, 1 spec.; (6569), 29.v.2008, 1 spec.; (6569), 14.vi.2008, 2 spec.

Halyzia sedecimguttata (Linnaeus, 1758): (6569), 22.vi.2008, 1 spec.

Hippodamia variegata (Goeze, 1777): (6569), 11.vii.2008, 4 spec.

Oenopia conglobata conglobata (Linnaeus, 1758): (6569), 14.vi.2008, 1 spec.

Propylea quatuordecimpunctata (Linnaeus, 1758): (6569), 9.v.2008, 2 spec.; (6569), 29.v.2008, 1 spec.; (6569), 22.vi.2008, 3 spec.; (6669), 22.vi.2008, 2 spec.

Psyllobora vigintiduopunctata (Linnaeus, 1758): (6569), 9.v.2008, 1 spec.; (6569), 29.v.2008, 2 spec.; (6569), 17.viii.2008, 1 spec.

Tytthaspis sedecimpunctata (Linnaeus, 1761): (6569), 22.vi.2008, 1 spec.; (6669), 22.vi.2008, 2 spec.

\section{CORYLOPHIDAE}

Colydium elongatum (Fabricius, 1787): (6569), 22.vi.2008, 1 spec., P. Průdek det.

Corylophus cassidoides (Marsham, 1802): (6569), 14.vi.2008, 1 spec.; (6569), 11.vii.2008, 1 spec.

Orthoperus atomus (Gyllenhal, 1808): (6569), 19.x.2008, 1 spec.

Orthoperus brunnipes (Gyllenhal, 1808): (6569), 19.x.2008, 9 spec.

Orthoperus corticalis (L. Redtenbacher, 1845): (6569), 19.x.2008, 2 spec.

Orthoperus punctatus Wankowicz, 1865: (6569), 19.x.2008, 1 spec.;

Sericoderus lateralis (Gyllenhal, 1827): (6569), 19.x.2008, 1 spec.

\section{LATRIDIIDAE}

Cartodere nodifer (Westwood, 1839): (6569), 9.v.2008, 1 spec.; (6569), 19.x.2008, 1 spec.

Corticaria serrata (Paykull, 1798): (6569), 11.vii.2008, 2 spec.

Corticarina minuta (Fabricius, 1792): (6569), 19.x.2008, 3 spec. 
Cortinicara gibbosa (Herbst, 1793): (6569), 29.v.2008, 2 spec.; (6569), 14.vi.2008, 1 spec.; (6569), 19.x.2008, 1 spec.

Enicmus histrio Joy et Tomlin, 1910: (6569), 19.x.2008, 2 spec.

Enicmus transversus transversus (Olivier, 1790): (6569), 19.x.2008, 2 spec.; (6669), 22.vi.2008, 1 spec.

Latridius consimilis (Mannerheim, 1844): (6569), 17.viii.2008, 1 spec.

Melanophthalma distinguenda (Comolli, 1837): (6569), 22.vi.2008, 1 spec.; (6569), 19.x.2008, 1 spec.

Melanophthalma transversalis (Gyllenhal, 1827): (6569), 29.v.2008, 1 spec.; (6569), 14.vi.2008, 1 spec.; (6569), 22.vi.2008, 1 spec.; (6569), 17.viii.2008, 2 spec.

Stephostethus angusticollis (Gyllenhal, 1827): (6569), 19.x.2008, 3 spec.

Stephostethus Iardarius (DeGeer, 1775): (6569), 19.x.2008, 5 spec.

\section{MYCETOPHAGIDAE}

Litargus balteatus LeConte, 1857: (6569), 17.viii.2008, 1 spec.

Litargus connexus (Geoffroy, 1785): (6569), 14.vi.2008, 2 spec.; (6569), 11.vii.2008, 2 spec.

Mycetophagus multipunctatus Fabricius, 1792: (6569), 14.vi.2008, 3 spec., O. Nakládal det.

Mycetophagus quadriguttatus P.W. J. Müller, 1821: (6569), 19.x.2008, 1 spec.

Triphyllus bicolor (Fabricius, 1777): (6569), 22.vi.2008, 1 spec., O. Nakládal det.

\section{CIIDAE}

Cis boleti (Scopoli, 1763): (6569), 19.x.2008, 1 spec.

Cis fagi Waltl, 1839: (6569), 19.x.2008, 2 spec.

Cis micans (Fabricius, 1792): (6569), 19.x.2008, 1 spec.

Cis rugulosus Mellié, 1848: (6569), 19.x.2008, 1 spec.

Octotemnus glabriculus (Gyllenhal, 1827): (6569), 19.x.2008, 3 spec.

Sulcacis fronticornis (Panzer, 1805): (6569), 19.x.2008, 5 spec.

Sulcacis nitidus (Fabricius, 1792): (6569), 17.viii.2008, 5 spec.

Sulcacis bidentulus (Rosenhauer, 1847): (6569), 19.x.2008, 1 spec.

\section{TETRATOMIDAE}

Tetratoma fungorum Fabricius, 1790: (6569), 19.x.2008, 1 spec.

\section{MELANDRYIDAE}

Anisoxya fuscula (Illiger, 1798): (6569), 14.vi.2008, 6 spec.

Hypulus bifasciatus (Fabricius, 1792): (6569), 9.v.2008, 1 spec., J. Vávra det.

Orchesia micans (Panzer, 1793): (6569), 9.v.2008, 1 spec.

\section{ZOPHERIDAE}

Aulonium trisulcum (Geoffroy 1785): (6569), 11.vii.2008, on light, 1 spec.

Bitoma crenata (Fabricius, 1775): (6569), 9.v.2008, 1 spec., P. Průdek det.; (6569), 11.vii.2008, 4 spec.

\section{MORDELLIDAE}

Mordella brachyura brachyura Mulsant, 1857: (6569), 14.vi.2008, 3 spec.; (6569), 22.vi.2008, 1 spec.; (6569), 11.vii.2008, 1 spec.; (6569), 17.viii.2008, 1 spec.

Mordellistena acuticollis Schilsky, 1895: (6569), 11.vii.2008, 1 spec.; (6569), 17.viii.2008, 2 spec.

Mordellistena humeralis (Fabricius, 1758): (6569), 14.vi.2008, 3 spec.; (6569), 22.vi.2008, 1 spec.

Mordellistena neuwaldeggiana (Panzer, 1796): (6569), 22.vi.2008, 1 spec.

Mordellistena parvula (Gyllenhal, 1827): (6569), 14.vi.2008, 1 spec.

Mordellistena pumila (Gyllenhal, 1810): (6569), 14.vi.2008, 4 spec.; (6569), 17.viii.2008, 1 spec.

Mordellistena secreta Horák, 1983: (6569), 14.vi.2008, 3 spec.

Mordellistena variegata (Fabricius, 1798): (6569), 22.vi.2008, 2 spec.; (6569), 11.vii.2008, on light, 1 spec.

Mordellistena weisei Schilsky, 1895: (6569), 14.vi.2008, 2 spec.

Mordellistena bicoloripilosa Ermisch, 1967: (6569), 14.vi.2008, 4 spec.; (6569), 22.vi.2008, 1 spec. 
Mordellistena inexpectata Ermisch, 1967: (6569), 14.vi.2008, 2 spec.

Mordellistena pseudoparvula Ermisch, 1956: (6669), 22.vi.2008, 1 spec.

Mordellochroa abdominalis (Fabricius, 1775): (6569), 29.v.2008, 6 spec.; (6669), 9.v.2008, 2 spec.

Variimorda villosa (Schrank, 1781): (6569), 11.vii.2008, 1 spec.

\section{TENEBRIONIDAE}

Allecula morio (Fabricius, 1787): (6569), 11.vii.2008, on light, 1 spec.

Alphitophagus bifasciatus (Say, 1824): (6569), 17.viii.2008, 1 spec.

Diaclina fagi (Panzer, 1799): (6569), 17.viii.2008, 2 spec.

Diaperis boleti (Linnaeus, 1758): (6569), 22.vi.2008, 3 spec.

Eledona agricola (Herbst, 1783): (6569), 14.vi.2008, 1 spec.; (6569), 22.vi.2008, 21 spec.

Lagria atripes Mulsant \& Guillebeau, 1855: (6569), 14.vi.2008, 1 spec., V. Švihla det.

Lagria hirta (Linnaeus, 1758): (6569), 11.vii.2008, 13 spec., V. Švihla det.

Pentaphyllus testaceus (Hellwig, 1792): (6569), 22.vi.2008, sifted from base of old tree, 2 spec.; (6569), 19.x.2008, 9 spec.

Stenomax aeneus Scopoli, 1763: (6569), 9.v.2008, 1 spec.; (6569), 29.v.2008, 1 spec.

\section{OEDEMERIDAE}

Ischnomera caerulea (Linnaeus, 1758): (6569), 29.v.2008, 1 spec.

Ischnomera cyanea (Fabricius, 1792): (6569), 9.v.2008, 2 spec.; (6569), 29.v.2008, 1 spec.

Oedemera femorata (Scopoli, 1763): (6569), 14.vi.2008, 2 spec.; (6569), 11.vii.2008, 2 spec.; (6569), 17.viii.2008, 1 spec.

Oedemera podagrariae podagrariae (Linnaeus, 1767): (6569), 17.viii.2008, 1 spec.

Oedemera virescens virescens (Linnaeus, 1767): (6569), 9.v.2008, 8 spec.; (6569), 29.v.2008, 2 spec.

\section{PYROCHROIDAE}

Pyrochroa coccinea (Linnaeus, 1761): (6569), 29.v.2008, 1 spec.

Pyrochroa serraticornis (Scopoli, 1763): (6569), 9.v.2008, 1 spec.; (6569), 29.v.2008, 1 spec.; (6669), 9.v.2008, $1 \mathrm{spec}$.

Schizotus pectinicornis (Linnaeus, 1758): (6569), 9.v.2008, 3 spec.

\section{SALPINGIDAE}

Lissodema cursor (Gyllenhal, 1813): (6569), 11.vii.2008, 1 spec.

Lissodema denticolle (Gyllenhal, 1813): (6569), 11.vii.2008, 2 spec.; (6569), 2008, under laboratory condition, 1 spec.

Salpingus planirostris (Fabricius, 1787): (6569), 9.v.2008, 2 spec.; (6569), 22.vi.2008, 1 spec.

\section{ANTHICIDAE}

Anthicus flavipes flavipes (Panzer, 1796): (6569), 17.viii.2008, 5 spec.

Anthicus sellatus (Panzer, 1796): (6569), 29.v.2008, 1 spec.; (6569), 14.vi.2008, 4 spec.; (6569), 17.viii.2008, 3 spec.; (6569), 19.x.2008, 1 spec.

Anthicus antherinus antherinus (Linnaeus, 1760): (6569), 9.v.2008, 2 spec.; (6569), 14.vi.2008, 1 spec.

Notoxus brachycerus (Faldermann, 1837): (6569), 14.vi.2008, 2 spec.; (6569), 22.vi.2008, 2 spec. (Kejval \& Nakládal 2009).

Notoxus monoceros (Linnaeus, 1760): (6569), 9.v.2008, 2 spec.; (6569), 29.v.2008, 7 spec.; (6569), 14.vi.2008, 2 spec.; (6569), 22.vi.2008, 1 spec.

Omonadus formicarius formicarius (Goeze, 1777): (6569), 17.viii.2008, 2 spec.

\section{SCRAPTIIDAE}

Anaspis flava (Linnaeus, 1758): (6569), 29.v.2008, 1 spec.; (6569), 14.vi.2008, 1 spec.

Anaspis frontalis (Linnaeus, 1758): (6569), 29.v.2008, 9 spec.; (6569), 14.vi.2008, 1 spec.

Anaspis varians varians Mulsant, 1857: (6569), 14.vi.2008, 2 spec. 


\section{CERAMBYCIDAE}

Agapanthia villosoviridescens (DeGeer, 1775): (6569), 14.vi.2008, 1 spec.

Alosterna tabacicolor tabacicolor (DeGeer, 1775): (6569), 29.v.2008, 3 spec.

Anaglyptus mysticus (Linnaeus, 1758): (6569), 9.v.2008, 1 spec.

Aromia moschata moschata (Linnaeus, 1758): (6569), 17.viii.2008, 1 spec.

Chlorophorus figuratus (Scopoli, 1763): (6569), 14.vi.2008, 2 spec.

Clytus arietis arietis (Linnaeus, 1758): (6569), 29.v.2008, 1 spec.; (6669), 9.v.2008, 1 spec.

Exocentrus adspersus Mulsant, 1846: (6569), 22.vi.2008, 1 spec.

Exocentrus punctipennis Mulsant et Guillebeau, 1857: (6569), 2008, under laboratory condition, 1 spec.

Gaurotes virginea virginea (Linnaeus, 1758): (6569), 29.v.2008, 4 spec.

Glaphyra umbellatarum (Schreber, 1759): (6569), 29.v.2008, 4 spec.; (6569), 22.vi.2008, 2 spec.

Grammoptera abdominalis (Stephens, 1831): (6669), 9.v.2008, 1 spec.

Grammoptera ruficornis ruficornis (Fabricius, 1781): (6569), 29.v.2008, 9 spec.

Grammoptera ustulata (Schaller, 1783): (6569), 9.v.2008, 2 spec.

Leiopus linnei Wallin, Nylander et Kvamme, 2009: (6569), 11.vii.2008, 1 spec.

Leptura annularis annularis Fabricius, 1801: (6569), 29.v.2008, 10 spec.

Leptura quadrifasciata quadrifasciata Linnaeus, 1758: (6569), 11.vii.2008, 2 spec.

Oberea linearis (Linnaeus, 1760): (6569), 29.v.2008, 1 spec.

Pachytodes cerambyciformis (Schrank, 1781): (6569), 14.vi.2008, 1 spec.

Phytoecia cylindrica (Linnaeus, 1758): (6569), 9.v.2008, 2 spec.; (6569), 29.v.2008, 3 spec.; (6569), 11.vii.2008, 1 spec.

Pidonia lurida (Fabricius, 1792): (6569), 29.v.2008, 3 spec.

Pogonocherus hispidus (Linnaeus, 1758): (6569), 9.v.2008, 1 spec.; (6569), 14.vi.2008, 1 spec.; (6569), 22.vi.2008, 1 spec.

Prionus coriarius (Linnaeus, 1758): (6569), 11.vii.2008, on light, 1 spec.

Pseudovadonia livida livida (Fabricius, 1777): (6569), 29.v.2008, 4 spec.; (6569), 14.vi.2008, 1 spec.

Rhagium mordax (DeGeer, 1775): (6569), 9.v.2008, 1 spec.; (6569), 14.vi.2008, 1 spec.

Rhagium sycophanta (Schrank, 1781): (6569), 9.v.2008, 2 spec.

Stenocorus meridianus (Linnaeus, 1758): (6569), 29.v.2008, 1 spec.

Stenostola dubia (Laicharting, 1784): (6569), 9.v.2008, 1 spec.; (6569), 29.v.2008, 1 spec.; (6569), 14.vi.2008, 1 spec.

Stenurella melanura (Linnaeus, 1758): (6569), 14.vi.2008, 3 spec.

Stictoleptura rubra rubra Linnaeus, 1758: (6569), 11.vii.2008, 1 spec.

Tetrops praeustus praeustus (Linnaeus, 1758): (6569), 29.v.2008, 1 spec.

\section{ORSODACNIDAE}

Orsodacne cerasi (Linnaeus, 1758): (6569), 29.v.2008, 1 spec.; (6569), 14.vi.2008, 1 spec.

Orsodacne humeralis humeralis Latreille, 1804: (6569), 9.v.2008, 2 spec.

\section{CHRYSOMELIDAE}

Altica lythri Aubé, 1843: (6569), 9.v.2008, 1 spec.

Aphthona euphorbiae (Schrank, 1781): (6569), 9.v.2008, 1 spec.; (6569), 29.v.2008, 1 spec.

Aphthona venustula (Kutschera, 1861): (6569), 9.v.2008, 1 spec.

Batophila rubi (Paykull, 1799): (6569), 14.vi.2008, 1 spec.

Bruchus atomarius (Linnaeus, 1760): (6569), 9.v.2008, 1 spec.

Cassida rubiginosa rubiginosa O. F. Müller, 1776: (6569), 9.v.2008, 1 spec.; (6569), 29.v.2008, 1 spec.; (6569), 14.vi.2008, 1 spec.; (6569), 22.vi.2008, 1 spec.

Cassida stigmatica Suffrian, 1844: (6569), 29.v.2008, 3 spec.

Cassida vibex Linnaeus, 1767: (6569), 9.v.2008, 1 spec.

Chaetocnema aridula (Gyllenhal, 1827): (6569), 9.v.2008, 5 spec.; (6569), 11.vii.2008, on light, 2 spec.; (6569), 17.viii.2008, 1 spec.; (6569), 19.x.2008, 1 spec.

Chaetocnema concinna (Marsham, 1802): (6569), 11.vii.2008, on light, 1 spec.

Chaetocnema hortensis (Geoffroy, 1785): (6569), 9.v.2008, 2 spec.; (6569), 11.vii.2008, 1 spec.; (6569), 17.viii.2008, 2 spec. 
Chaetocnema mannerheimii (Gyllenhal, 1827): (6569), 29.v.2008, 1 spec.; (6569), 22.vi.2008, 2 spec.; (6569), 11.vii.2008, 1 spec.

Chaetocnema picipes Stephens, 1831: (6569), 9.v.2008, 1 spec.; (6569), 17.viii.2008, 1 spec.

Chrysolina fastuosa fastuosa (Scopoli, 1763): (6569), 29.v.2008, 1 spec.; (6569), 14.vi.2008, 2 spec.; (6569), 11.vii.2008, 1 spec.

Chrysolina graminis graminis (Linnaeus, 1758): (6569), 17.viii.2008, 1 spec.

Chrysolina herbacea herbacea (Duftschmid, 1825): (6569), 11.vii.2008, 1 spec.

Chrysolina oricalcia (O. F. Müller, 1776): (6569), 22.vi.2008, 1 spec.

Chrysolina sturmi (Westhoff, 1882): (6569), 14.vi.2008, 1 spec.

Chrysolina varians (Schaller, 1783): (6569), 14.vi.2008, 1 spec.

Chrysomela populi Linnaeus, 1758: (6569), 11.vii.2008, 5 spec.

Clytra laeviuscula Ratzeburg, 1837: (6569), 22.vi.2008, 3 spec.; (6569), 11.vii.2008, 3 spec.

Crepidodera aurata (Marsham, 1802): (6569), 9.v.2008, 1 spec.; (6569), 29.v.2008, 2 spec.; (6569), 14.vi.2008, 2 spec.

Crepidodera aurea (Geoffroy, 1785): (6569), 9.v.2008, 1 spec.; (6569), 29.v.2008, 9 spec.; (6569), 14.vi.2008, 1 spec.; (6669), 22.vi.2008, 1 spec.

Crepidodera plutus (Latreille, 1804): (6569), 14.vi.2008, 1 spec.

Cryptocephalus bilineatus (Linnaeus, 1767): (6569), 22.vi.2008, 1 spec.

Cryptocephalus moraei (Linnaeus, 1758): (6569), 14.vi.2008, 1 spec.; (6569), 22.vi.2008, 6 spec.; (6669), 22.vi.2008, 1 spec.

Cryptocephalus ocellatus ocellatus Drapiez, 1819: (6569), 29.v.2008, 5 spec.; (6569), 22.vi.2008, 2 spec.

Cryptocephalus populi Suffrian, 1848: (6569), 17.viii.2008, 1 spec.

Epitrix pubescens (Koch, 1803): (6569), 29.v.2008, 1 spec.

Galerucella lineola lineola (Fabricius, 1781): (6569), 9.v.2008, 1 spec.; (6569), 29.v.2008, 2 spec.

Gastrophysa viridula viridula (DeGeer, 1775): (6569), 14.vi.2008, 2 spec.

Gonioctena quinquepunctata quinquepunctata (Fabricius, 1787): (6569), 9.v.2008, 1 spec.

Hispa atra Linnaeus, 1767: (6569), 22.vi.2008, 1 spec.

Labidostomis longimana (Linnaeus, 1760): (6569), 11.vii.2008, 4 spec.; (6669), 22.vi.2008, 1 spec.

Lema cyanella (Linnaeus, 1758): (6669), 22.vi.2008, 1 spec.

Lilioceris merdigera (Linnaeus, 1758): (6569), 9.v.2008, 1 spec.; (6569), 29.v.2008, 3 spec.; (6569), 11.vii.2008, 1 spec.

Longitarsus anchusae (Paykull, 1799): (6569), 9.v.2008, 2 spec.; (6569), 14.vi.2008, 5 spec.; (6569), 22.vi.2008, 2 spec.; (6669), 22.vi.2008, 3 spec.

Longitarsus linnaei (Duftschmid, 1825): (6569), 29.v.2008, 2 spec.; (6569), 14.vi.2008, 6 spec.

Longitarsus luridus luridus (Scopoli, 1763): (6569), 9.v.2008, 1 spec.

Longitarsus lycopi (Foudras, 1860): (6569), 9.v.2008, 1 spec.

Longitarsus melanocephalus (DeGeer, 1775): (6569), 9.v.2008, 1 spec.; (6569), 14.vi.2008, 1 spec.; (6569), 22.vi.2008, 1 spec.

Longitarsus monticola Kutschera, 1864: (6569), 9.v.2008, 1 spec.

Longitarsus nigrofasciatus nigrofasciatus (Goeze, 1777): (6569), 14.vi.2008, 1 spec.

Longitarsus rubiginosus (Foudras, 1860): (6569), 17.viii.2008, 1 spec.

Longitarsus suturellus (Duftschmid, 1825): (6569), 14.vi.2008, 2 spec.

Neocrepidodera ferruginea (Scopoli, 1763): (6569), 22.vi.2008, 1 spec.; (6569), 11.vii.2008, 1 spec.; (6569), 17.viii.2008, 3 spec.

Oomorphus concolor (Sturm, 1807): (6569), 14.vi.2008, 5 spec.

Oulema gallaeciana Heyden, 1870: (6569), 9.v.2008, 2 spec.; (6569), 9.v.2008, 1 spec.; (6569), 29.v.2008, 2 spec.; (6569), 14.vi.2008, 1 spec.; (6569), 22.vi.2008, 2 spec.; (6569), 11.vii.2008, 2 spec.; (6569), 17.viii.2008, 5 spec.; (6569), 19.x.2008, 1 spec.

Oulema melanopus (Linnaeus, 1758): (6569), 22.vi.2008, 1 spec.; (6569), 11.vii.2008, 1 spec.

Pachybrachis hippophaes Suffrian, 1848: (6569), 14.vi.2008, 1 spec.

Phaedon cochleariae cochleariae (Fabricius, 1792): (6569), 14.vi.2008, 1 spec.; (6569), 22.vi.2008, 1 spec.

Phyllotreta atra (Fabricius, 1775): (6569), 29.v.2008, 1 spec.; (6569), 22.vi.2008, 1 spec.; (6569), 11.vii.2008, 1 spec.; (6569), 19.x.2008, 1 spec.; (6669), 22.vi.2008, 1 spec.

Phyllotreta cruciferae (Goeze, 1777): (6569), 9.v.2008, 1 spec.

Phyllotreta nemorum (Linnaeus, 1758): (6569), 29.v.2008, 3 spec.; (6569), 14.vi.2008, 3 spec.; (6569), 22.vi.2008, 2 spec.

Phyllotreta nigripes nigripes (Fabricius, 1775): (6569), 11.vii.2008, $1 \mathrm{spec}$.

Phyllotreta ochripes (Curtis, 1837): (6569), 9.v.2008, 1 spec.; (6569), 29.v.2008, 1 spec.; (6569), 14.vi.2008, 2 spec.; (6569), 22.vi.2008, 1 spec.; (6569), 11.vii.2008, 1 spec. 
Phyllotreta striolata (Illiger, 1803): (6569), 9.v.2008, 1 spec.; (6569), 22.vi.2008, 2 spec.; (6569), 11.vii.2008, 1 spec.

Phyllotreta undulata (Kutschera, 1860): (6569), 14.vi.2008, 1 spec.; (6569), 22.vi.2008, 3 spec.; (6669), 22.vi.2008, 1 spec.

Phyllotreta vittula (L. Redtenbacher, 1849): (6569), 9.v.2008, 2 spec.; (6569), 29.v.2008, 1 spec.

Psylliodes dulcamarae (Koch, 1803): (6569), 17.viii.2008, 1 spec.

Psylliodes napi (Fabricius, 1792): (6569), 14.vi.2008, 1 spec.

Smaragdina affinis affinis (Illiger, 1794): (6569), 9.v.2008, 4 spec.; (6569), 29.v.2008, 6 spec.

Sphaeroderma testaceum (Fabricius, 1775): (6669), 22.vi.2008, 2 spec.

\section{ANTHRIBIDAE}

Anthribus nebulosus Forster, 1771: (6569), 9.v.2008, 2 spec.; (6669), 9.v.2008, 1 spec.

Platystomos albinus (Linnaeus, 1758): (6569), 9.v.2008, 1 spec., O. Nakládal det.; (6569), 14.vi.2008, 1 spec., O. Nakládal det.

Rhaphitropis marchicus (Herbst, 1797): (6569), 29.v.2008, 1 spec.

\section{ATTELABIDAE}

Caenorhinus aequatus (Linnaeus, 1767): (6569), 9.v.2008, 1 spec.; (6669), 9.v.2008, 1 spec.

Caenorhinus germanicus (Herbst, 1797): (6569), 9.v.2008, 1 spec.

\section{APIONIDAE}

Apion cruentatum Walton, 1844: (6569), 9.v.2008, 2 spec.

Apion frumentarium (Linnaeus, 1758): (6569), 9.v.2008, 1 spec.; (6569), 14.vi.2008, 2 spec.; (6669), 22.vi.2008, 1 spec.

Betulapion simile (W. Kirby, 1811): (6569), 9.v.2008, 1 spec.; (6569), 19.x.2008, 1 spec.

Catapion pubescens (W. Kirby, 1811): (6569), 22.vi.2008, 1 spec.

Ceratapion onopordi (W. Kirby, 1808): (6569), 9.v.2008, 1 spec.; (6569), 29.v.2008, 1 spec.; (6569), 14.vi.2008, 5 spec.; (6569), 22.vi.2008, 3 spec.; (6569), 17.viii.2008, 2 spec.; (6669), 22.vi.2008, 3 spec.

Diplapion confluens (W. Kirby, 1808): (6569), 17.viii.2008, 1 spec.

Eutrichapion viciae (Paykull, 1800): (6569), 9.v.2008, 1 spec.; (6569), 29.v.2008, 1 spec.; (6569), 17.viii.2008, 5 spec.

Ischnopterapion virens (Herbst, 1797): (6569), 14.vi.2008, 1 spec.; (6569), 22.vi.2008, 1 spec.

Protapion nigritarse (W. Kirby, 1808): (6569), 9.v.2008, 1 spec.; (6569), 29.v.2008, 3 spec.; (6569), 22.vi.2008, 1 spec.; (6569), 19.x.2008, 1 spec.

Protapion trifolii (Linnaeus, 1768): (6569), 29.v.2008, 2 spec.

Rhopalapion longirostre (Olivier, 1807): (6569), 9.v.2008, 1 spec.

Squamapion cineraceum (Wencker, 1864): (6569), 9.v.2008, 1 spec.; (6569), 17.viii.2008, 2 spec.

Taeniapion urticarium (Herbst, 1784): (6569), 11.vii.2008, 1 spec.; (6669), 22.vi.2008, 1 spec.

\section{CURCULIONIDAE}

Acalles echinatus (Germar, 1824): (6569), 14.vi.2008, 1 spec.; (6569), 19.x.2008, 10 spec.

Acalyptus carpini (Fabricius, 1792): (6569), 9.v.2008, 5 spec.

Alophus triguttatus triguttatus (Fabricius, 1775): (6569), 9.v.2008, 1 spec.; (6569), 9.v.2008, 2 spec.; (6569), 14.vi.2008, 1 spec.; (6569), 29.v.-11.vii.2008, PFT - locality 3, 1 spec.

Anthonomus humeralis (Panzer, 1795): (6569), 29.v.2008, 22 spec.; (6569), 14.vi.2008, 1 spec.

Anthonomus pomorum (Linnaeus, 1758): (6569), 29.v.2008, 5 spec.; (6569), 14.vi.2008, 1 spec.; (6569), 22.vi.2008, 1 spec.

Anthonomus rectirostris (Linnaeus, 1758): (6569), 9.v.2008, 1 spec.; (6569), 11.vii.2008, 1 spec.

Anthonomus rubi (Herbst, 1795): (6569), 29.v.2008, 1 spec.; (6569), 14.vi.2008, 1 spec.

Archarius crux (Fabricius, 1776): (6569), 9.v.2008, 1 spec.

Baris artemisiae (Herbst, 1795): (6569), 29.v.2008, 1 spec.

Barypeithes chevrolati (Boheman, 1843): (6569), 22.vi.2008, 5 spec.

Bradybatus kellneri Bach, 1854: (6569), 22.vi.2008, 1 spec.; (6569), 19.x.2008, 1 spec. 
Ceutorhynchus erysimi (Fabricius, 1787): (6569), 9.v.2008, 1 spec.; (6569), 14.vi.2008, 4 spec.; (6569), 22.vi.2008, 1 spec.; (6669), 22.vi.2008, 1 spec.

Ceutorhynchus floralis (Herbst, 1795): (6569), 9.v.2008, 1 spec.; (6569), 29.v.2008, 1 spec.; (6569), 14.vi.2008, 1 spec.; (6569), 22.vi.2008, 1 spec.; (6669), 22.vi.2008, 1 spec.

Ceutorhynchus interjectus Schultze, 1903: (6569), 14.vi.2008, 5 spec.

Ceutorhynchus obstrictus (Marsham, 1802): (6569), 9.v.2008, 1 spec.

Ceutorhynchus pallidactylus (Marsham, 1802): (6569), 22.vi.2008, 1 spec.; (6569), 19.x.2008, 3 spec.

Ceutorhynchus pectoralis Weise, 1895: (6569), 14.vi.2008, 1 spec.

Ceutorhynchus scrobicollis Neresheimer et Wagner, 1924: (6569), 14.vi.2008, $1 \mathrm{spec}$.

Chlorophanus viridis (Linnaeus, 1758): (6569), 22.vi.2008, 5 spec.

Cionus tuberculosus (Scopoli, 1763): (6569), 9.v.2008, 2 spec.; (6569), 22.vi.2008, 1 spec.

Coeliastes lamii (Fabricius, 1792): (6569), 9.v.2008, 1 spec.; (6569), 29.v.2008, 5 spec.

Coeliodes erythroleucos (Gmelin, 1790): (6569), 29.v.2008, 1 spec.

Coryssomerus capucinus (Beck, 1817): (6569), 9.v.2008, 1 spec.; (6569), 9.v.2008, 1 spec.

Curculio glandium Marsham, 1802: (6569), 9.v.2008, 1 spec.

Curculio pellitus (Boheman, 1843): (6569), 9.v.2008, 1 spec.

Curculio venosus (Gravenhorst, 1807): (6569), 9.v.2008, 2 spec.; (6569), 29.v.2008, 1 spec.

Datonychus arquatus (Herbst, 1795): (6569), 14.vi.2008, 3 spec.

Dorytomus filirostris (Gyllenhal, 1836): (6569), 29.v.2008, 1 spec.

Dorytomus longimanus (Forster, 1771): (6569), 29.v.2008, 22 spec.; (6569), 19.x.2008, 1 spec.

Dorytomus nebulosus (Gyllenhal, 1836): (6569), 9.v.2008, 2 spec.; (6569), 29.v.2008, 1 spec.; (6569), 22.vi.2008, 1 spec.; (6669), 9.v.2008, 2 spec.

Dorytomus puberulus (Boheman, 1843): (6569), 14.vi.2008, 2 spec.; (6669), 9.v.2008, 1 spec.

Dorytomus suratus (Gyllenhal, 1836): (6569), 22.vi.2008, 1 spec.

Dorytomus tremulae (Fabricius, 1787): (6569), 9.v.2008, 1 spec.; (6569), 29.v.2008, 2 spec.

Ellescus infirmus (Herbst, 1795): (6569), 29.v.2008, 1 spec.

Ellescus scanicus (Paykull, 1792): (6569), 14.vi.2008, 1 spec.

Eusomus ovulum Germar, 1824: (6569), 9.v.2008, 2 spec.; (6569), 29.v.2008, 1 spec.; (6569), 22.vi.2008, 1 spec.; (6669), 22.vi.2008, 2 spec.

Hadroplontus trimaculatus (Fabricius, 1775): (6669), 22.vi.2008, 2 spec.

Hylesinus crenatus (Fabricius, 1787): (6569), 2008, under laboratory condition, 3 spec.

Hylobius abietis (Linnaeus, 1758): (6569), 9.v.2008, 1 spec.

Hypera meles (Fabricius, 1792): (6569), 9.v.2008, 1 spec.; (6569), 22.vi.2008, 1 spec.; (6569), 11.vii.2008, on light, 1 spec.

Hypera rumicis (Linnaeus, 1758): (6569), 29.v.2008, 1 spec.

Larinus jaceae (Fabricius, 1775): (6569), 22.vi.2008, 1 spec.; (6669), 22.vi.2008, 1 spec.

Larinus planus (Fabricius, 1792): (6569), 9.v.2008, 1 spec.

Lepyrus palustris (Scopoli, 1763): (6569), 11.vii.2008, 1 spec.

Lignyodes muerlei Ferrari, 1886: (6569), 29.v.2008, 1 spec.

Lignyodes uniformis Desbrochers, 1894: (6569), 9.v.2008, 2 spec.; (6569), 29.v.2008, 4 spec.; (6569), 14.vi.2008, $1 \mathrm{spec}$.

Liophloeus lentus Germar, 1824: (6569), 9.v.2008, 2 spec.; (6569), 29.v.2008, 1 spec.; (6569), 14.vi.2008, 2 spec.

Liparus glabrirostris Küster, 1849: (6569), 9.v.2008, 5 spec.; (6569), 14.vi.2008, 12 spec.

Magdalis armigera (Geoffroy, 1785): (6569), 29.v.2008, 1 spec.

Mecinus labilis (Herbst, 1795): (6569), 9.v.2008, 1 spec.

Microplontus rugulosus (Herbst, 1795): (6569), 9.v.2008, 1 spec.

Mogulones larvatus (Schultze, 1896): (6569), 9.v.2008, 1 spec.

Nedyus quadrimaculatus (Linnaeus, 1758): (6569), 9.v.2008, 2 spec.; (6569), 29.v.2008, 3 spec.; (6569), 14.vi.2008, 6 spec.; (6669), 22.vi.2008, 1 spec.

Otiorhynchus ovatus (Linnaeus, 1758): (6569), 17.viii.2008, $1 \mathrm{spec}$.

Otiorhynchus raucus (Fabricius, 1777): (6569), 14.vi.2008, 1 spec.; (6569), 11.vii.-17.viii.2008, PFT - locality 1, 2 spec.; (6569), 29.v.-11.vii.2008, PFT - locality 2, 2 spec.; (6569), 29.v.-11.vii.2008, PFT - locality 4, 1 spec.; (6569), 29.v.-11.vii.2008, PFT - locality 1, 1 spec.

Parethelcus pollinarius (Forster, 1771): (6569), 9.v.2008, 1 spec.; (6569), 29.v.2008, 1 spec.; (6569), 22.vi.2008, 1 spec.

Phyllobius glaucus (Scopoli, 1763): (6569), 29.v.2008, 2 spec.; (6569), 14.vi.2008, 1 spec.

Phyllobius oblongus (Linnaeus, 1758): (6569), 9.v.2008, 1 spec.; (6569), 29.v.2008, 3 spec.; (6569), 14.vi.2008, 1 spec. 
Phyllobius pomaceus Gyllenhal, 1834: (6569), 9.v.2008, 5 spec.; (6569), 29.v.2008, 2 spec.; (6569), 14.vi.2008, 1 spec.

Phyllobius pyri (Linnaeus, 1758): (6569), 9.v.2008, 2 spec.; (6569), 29.v.2008, 3 spec.; (6669), 9.v.2008, 1 spec. Phyllobius vespertinus (Fabricius, 1792): (6569), 9.v.2008, 2 spec.

Phyllobius virideaeris (Laicharting, 1781): (6569), 9.v.2008, 3 spec.; (6569), 29.v.2008, 1 spec.

Polydrusus pterygomalis Boheman, 1840: (6569), 9.v.2008, 1 spec.

Polydrusus sericeus (Schaller, 1783): (6569), 29.v.2008, 8 spec.; (6569), 14.vi.2008, 2 spec.

Rhamphus pulicarius (Herbst, 1795): (6569), 29.v.2008, 1 spec.

Rhinoncus bruchoides (Herbst, 1784): (6569), 11.vii.2008, 1 spec.

Rhinoncus pericarpius (Linnaeus, 1758): (6569), 9.v.2008, 3 spec.; (6569), 29.v.2008, 2 spec.; (6569), 14.vi.2008, 6 spec.; (6569), 22.vi.2008, 1 spec.

Rhinusa bipustulata (Rossi, 1792): (6569), 14.vi.2008, 1 spec.

Rhynchaenus populicola Silfverberg, 1977: (6569), 14.vi.2008, 1 spec.

Rhynchaenus salicis (Linnaeus, 1758): (6669), 9.v.2008, 1 spec.

Rhyncolus punctatulus (Boheman, 1838): (6569), 2008, under laboratory condition, 6 spec.

Sciaphilus asperatus (Bonsdorff, 1785): (6569), 9.v.2008, 3 spec.

Scolytus intricatus (Ratzeburg, 1837): (6569), 11.vii.2008, 1 spec.; (6569), 11.vii.2008, on light, 1 spec.

Scolytus mali (Bechstein, 1805): (6569), 17.viii.2008, only galleries

Scolytus multistriatus (Marsham, 1802): (6569), 11.vii.2008, 1 spec.

Scolytus ratzeburgii Janson, 1857: (6569), 17.viii.2008, only galleries

Sitona hispidulus (Fabricius, 1776): (6569), 9.v.2008, 2 spec.

Sitona humeralis Stephens, 1831: (6569), 19.x.2008, 1 spec.

Sitona languidus Gyllenhal, 1834: (6569), 14.vi.2008, 2 spec.

Sitona lepidus Gyllenhal, 1834: (6569), 17.viii.2008, 1 spec.

Sitona lineatus (Linnaeus, 1758): (6569), 9.v.2008, 3 spec.; (6569), 17.viii.2008, 3 spec.

Sitona sulcifrons (Thunberg, 1798): (6569), 9.v.2008, 1 spec.

Sitophilus oryzae (Linnaeus, 1758): (6569), 17.viii.2008, 1 spec.

Stenocarus ruficornis (Stephens, 1831): (6569), 11.vii.2008, 1 spec.

Tanymecus palliatus (Fabricius, 1787): (6569), 9.v.2008, 5 spec.; (6569), 11.vii.2008, 2 spec.

Trachodes hispidus (Linnaeus, 1758): (6569), 11.vii.2008, 1 spec.; (6569), 19.x.2008, 2 spec.

Trichosirocalus hassicus (Schultze, 1901): (6569), 9.v.2008, 4 spec.; (6569), 22.vi.2008, 3 spec.

Trichosirocalus troglodytes (Fabricius, 1787): (6569), 9.v.2008, 2 spec.; (6569), 22.vi.2008, 4 spec.; (6569), 17.viii.2008, 2 spec.

Tropiphorus elevatus (Herbst, 1795): (6569), 9.v.2008, 1 spec.

Tychius picirostris (Fabricius, 1787): (6569), 29.v.2008, 1 spec.; (6569), 22.vi.2008, 1 spec.; (6669), 9.v.2008, 1 spec.

\section{Dicussion}

During the all survey 1 species belonging to the NATURA 2000 was recorded, next 5 species are protected by law in the Czech Republic, 4 species are critically endangered according to Red list of theatened species in the Czech Republic (abbreviated as RL) (Farkač et al. 2005), 15 endangered (RL), 15 near theatened (RL), 32 vulnerable (RL). In following text, only species with faunistic data presenting in this paper are listed.

Vulnerable (according RL): Anisoxya fuscula (Melandryidae), Aulonium trisulcum (Zopheridae), Lathropus sepicola (Laemophloeidae), Mycetophagus multipunctatus (Mycetophagidae), Pentaphyllus testaceus (Tenebrionidae), Scolytus multistriatus (Curculionidae), Sulcacis bidentulus (Ciidae) and Triphyllus bicolor (Mycetophagidae).

Near theatened (according RL): Acalles echinatus, Ceutorhynchus pectoralis, Trachodes hispidus (Curculionidae), Aromia moschata and Rhagium sycophanta (Cerambycidae).

Endangered (according RL): Anthicus sellatus (Anthicidae), Cerylon deplanatum (Cerylonidae), Cucujus cinnaberinus (Cucujidae), Diaclina fagi (Tenebrionidae), Oomorphus concolor (Chrysomelidae) and Oxylaemus cylindricus (Bothrideridae).

Critically endangered (according RL): Longitarsus linnaei (Chrysomelidae) is stenotopic monophagous species with Symphytum tuberosum as host plant. It occurred from lowlands to uplands with activity from April end till July beginning (maximum in May). The species was 
known in the Czech Republic only from southern Moravia (local and rare there) (Čížek \& Douget 2008).

NATURA 2000: Cucujus cinnaberinus (Cucujidae).

\section{Conclusions}

Inventory survey of beetles (Coleoptera) was performed in Zástudánčí NNR. NNR is sited in Central Moravia by the southeastern from Tovačov town. 3585 specimens belonging to 857 species from 64 families were recorded during all survey (Nakládal 2011 and this paper). Faunistic data of 41 of them (Nosodendridae, Dermestidae, Anobidae, Cleridae, Melyridae, Sphindidae, Kateretidae, Nitidulidae, Monotomidae, Silvanidae, Cucujidae, Laemophloeidae, Phalacridae, Cryptophagidae, Erotylidae, Byturidae, Bothrideridae, Cerylonidae, Endomychidae, Coccinellidae, Colydiidae, Corylophidae, Latridiidae, Mycetophagidae, Ciidae, Tetratomidae, Melandryidae, Mordellidae, Tenebrionidae, Oedemeridae, Pyrochroidae, Salpingidae, Anthicidae, Scraptiidae, Cerambycidae, Orsodacnidae, Chrysomelidae, Anthribidae, Attelabidae, Apionidae, and Curculionidae) are presented here. Presenting part of data represents 1394 specimens belonging to 401 species.

During the all survey (Nakládal 2011 and this paper) one recorded species belong to the NATURA 2000, another 4 species are endangered according to notice of Ministry of the Environment of the Czech Republic no. 395/1992 code of law. Four critically endangered, 15 endangered, 15 near threatened and 32 vulnerable species according Red list of threatened species in the Czech Republic (Farkač et al. 2005) were also recorded.

Majority of these interesting species are associate to old tree stands or only to single old trees (very old succession stage) or conversely on very early succession stages like sandy and gravely riparian zone along the Morava river are. Management of nature preservation should be evolved from these findings. Riparian zone is not endangered (in case conserving natural river dynamics), old trees particularly on dam (beyond the northwestern boundary of Zástudánčí National Nature Reserve) can be endangered by direct liquidation in the future. Just this part is very valuable in nature preservation point of view (Nakládal 2011).

Acknowledgement: Thanks belong to all those who provided me identification of some groups of beetles. This paper was written in the framework of the research project NAZV 81136 'Survey of optimal methods used as protection measures against Ips typographus (L.) in different outbreak levels‘.

\section{References}

Čelechovská J. (in litt.): Denní motýli (Lepidoptera: Rhopalocera) v okolí Tovačova. (Diplomová práce). [Daily butterfly (Lepidoptera: Rhopalocera) of Central Moravia region] (Diploma thesis)]. Faculty of Science, Palacký University Olomouc, 2001, 73 pp., Olomouc.

Čelechovský A. (2001b): Motýli (Macrolepidoptera) NPR Zástudánčí u Tovačova. Macrolepidoptera of the Zástudánčí Nature Reserve near Tovačov. Př́rodověd. Stud. Muz. (Prostějov), 4: 103-114.

- (in litt.): Píd'alkovití (Geometridae, Lepidoptera) okolí Tovačova. (Diplomová práce). [Geometridae (Lepidoptera) of Tovačov town environs] (Unpublished diploma thesis). Faculty of Science, Palacký University Olomouc, 1993, 112 pp., Olomouc.

- (in litt.): Denní motýli (Lepidoptera: Rhopalocera) na území střední Moravy. (Disertační práce). [Daily butterfly (Lepidoptera: Rhopalocera) of Central Moravia region] (Ph.D. thesis). Faculty of Science, Palacký University Olomouc, 2001a, 130 pp., Olomouc.

Čížek P. \& Doguet S. (2008): Klíč k určování dřepčíků (Coleoptera: Chrysomelidae: Alticinae) Česka a Slovenska. [Key for the identification of the flea beetles (Coleoptera: Chrysomelidae: Alticinae) of the Czech and Slovak Republics]. Městské muzeum, Nové Město nad Metují, 232 pp.

Farkač J., Král D. \& Šk korpík M. (2005): Červený seznam ohrožených druhů České Republiky - Bezobratlí. (Red list of threatened species in the Czech Republic - Invertebrates). 758 pp., AOPK, Praha.

Kejval Z. \& Nakládal O. (2009): Faunistic records from Czech Republic - 275. Coleoptera: Anthicidae. Klapalekiana, 45 (1-2): 117-118.

Löbl I. \& Smetana A. (eds) (2007): Catalogue of Palaearctic Coleoptera, Vol. 4: Elateroidea - Derodontoidea - Bostrichoidea - Lymexyloidea - Cleroidea - Cucujoidea. Apollo Books, Stenstrup, 935 pp.

- (eds) (2008): Catalogue of Palaearctic Coleoptera, Vol. 5: Tenebrionoidea. Apollo Books, Stenstrup, 670 pp.

- (eds) (2010): Catalogue of Palaearctic Coleoptera, Vol. 6: Chrysomeloidea. Apollo Books, Stenstrup, 924 pp.

Merta L. (in litt.): Inventarizační průzkum NPR Zástudánčí z oboru hydrobiologie. (Závěrečná zpráva), [Hydrobiology Inventory study of the Zástudánčí National Nature Reserve]. Unpubl. manuscript, deposited at the Administration of the Litovelské Pomoraví PLA, 2004: 11 pp.

Nakládal O. (2011): Results of beetles (Coleoptera) survey of Zástudánčí National Nature Reserve (Central Moravia) 2008 - part 1. Čas. Slez. Muz. Opava (A), 60: 63-78. 
Pruner L. \& Míka P. (1996): Seznam obcí a jejich částí v České republice s čísly mapových polí pro sítové mapování fauny. (List of settlements in the Czech Republic with associated map field codes for faunistic grid mapping system). Klapalekiana 32 (Suppl.): 1-115.

Šafář J. et al. (2003): Olomoucko [Olomouc region]. In: Mackovčin P., Sedláček M. (eds.): Chráněná území ČR, svazek VI, [Protected areas of the Czech Republic, volume VI]. 456 pp., Agentura ochrany př́rody a krajiny ČR a EkoCentrum Brno, Praha.

Vorst O. (ed.) (2010): Catalogus van de Nederlandse kevers. (Catalogue of the Coleoptera of the Netherlands). Monografieën van de Nederlandse Entomologische Vereniging 11: 1-317 pp. + CD. (in Dutch, English Introduction and Summary).

\section{Výsledky průzkumu brouků (Coleoptera) NPR Zástudánčí (střední Morava) provedeného v roce 2008 - 2. část}

V NPR Zástudánčí, která se nachází na střední Moravě jihovýchodně od města Tovačov byl v roce 2008 proveden inventarizační průzkum brouků (Coleoptera). S cílem získat maximální druhovou diverzitu zkoumaného území bylo různými metodami odchyceno celkem 3585 exemplářu. Odchycené exempláře představují 857 druhů ze 64 čeledí. Tento příspěvek prezentuje faunistická data k 1394 exemplářum, které představují 401 druhů ze 41 čeledí.

Z celkového počtu zaznamenaných druhů je 1 druh zařazen do soustavy NATURA 2000, dle př. č.III. vyhl. MŽP ČR č. 395/1992 Sb. bylo nalezeno 5 ohrožených druhů, dle Červeného seznamu (Farkač et al. 2005) (dále jen ČS) 4 druhy kriticky ohrožené, 15 druhů ohrožených, 15 téměř ohrožených a 32 zranitelných.

Většina zajímavých druhů (řazených do systému NATURA 2000, do př. č.III. vyhl. MŽP ČR č. 395/1992 Sb. a do ČS) je vázána na staré porosty či jednotlivé staré stromy, čili velmi stará sukcesní stádia lesa, nebo naopak na velmi mladá sukcesní stádia typu př́břežních štěrkopískových lavic při březích řeky Moravy. Od tohoto by se měl odvíjet i management ochrany přírody. Zatímco příbřežní porosty nejsou při zachování přirozené dynamiky řeky ohroženy, staré stromy při severozápadní hranici NPR (mapka viz Nakládal (2011), rostoucí na hrázi (mimo NPR) mohou být $\mathrm{v}$ budoucnu ohroženy prímou likvidací. Přitom právě tato hráz je $\mathrm{z}$ hlediska chrany př́rody velmi cenná a zasluhuje zvláštní pozornost (Nakládal 2011).

Author's address: Oto Nakládal, Department of Forest Protection and Game Management, Faculty of Forestry and Wood Sciences, Czech University of Life Sciences Prague, Kamýcká 129, CZ-165 21 Praha 6 - Suchdol, Czech Republic; e-mail: nakladal@fld.czu.cz 